\title{
Gravitational collapse of rotating spheroids and the formation of naked singularities
}

\author{
Stuart L. Shapiro and Saul A. Teukolsky \\ Center for Radiophysics and Space Research, and Departments of Astronomy and Physics, \\ Cornell University, Ithaca, New York 14853
}

(Received 26 September 1991)

\begin{abstract}
We explore numerically the effect of rotation on the collapse of collisionless gas spheroids in full general relativity. The spheroids are initially prolate and consist of equal numbers of corotating and counterrotating particles. We have previously shown that in the absence of rotation the spheroids all collapse to spindle singularities. When the spheroids are sufficiently compact, the singularities are hidden inside black holes. However, when the spheroids are large enough, there are no apparent horizons. These nonrotating spheroids are strong candidates for naked singularities. Here our simulations suggest that rotation significantly modifies the evolution when it is sufficiently large. Imploding configurations with appreciable rotation ultimately collapse to black holes. However, for small enough angular momentum, our simulations cannot at present distinguish rotating from nonrotating collapse: spindle singularities appear to arise without apparent horizons. Hence it is possible that even spheroids with some angular momentum may form naked singularities.
\end{abstract}

PACS number(s): 04.20.Jb, 95.30.Sf, 97.60.Lf

\section{INTRODUCTION}

We recently reported $[1,2]$ results of numerical calculations that identified strong candidates for the formation of naked singularities in general relativity. We followed the gravitational collapse of oblate and prolate collisionless gas spheroids from rest. The spheroids satisfied the relativistic Vlasov equation for the matter coupled to Einstein's equations for the gravitational field. These simulations were the relativistic generalizations of LinMestel-Shu [3] homogeneous spheroid collapse in Newtonian theory. Our numerical code was designed to treat highly aspherical gravitational collapse in axisymmetry. We found that prolate spheroids collapse to spindle singularities. When the spheroids are sufficiently compact, the singularities are hidden inside black holes. However, when the spheroids are sufficiently large, there are no apparent horizons. These results lend support to the hoop conjecture [4] and provide evidence that naked singularities can form in asymptotically flat spacetimes.

In all the examples described above, the particles were initially at rest and hence they had no angular momentum. It is known that in Newtonian gravitation the presence of angular momentum, however small, prevents an infinitesimally thin spindle singularity from forming on the symmetry axis. It is also known [5] that in Newtonian theory the singularity that forms during homogeneous Lin-Mestel-Shu collapse can be prevented by any slight initial inhomogeneity or velocity dispersion. It is not at all obvious that the same results hold in general relativity, where the gravitational field is nonlinear. For example, in spherical relativistic collapse we know from countless numerical simulations that small perturbations are not sufficient to prevent singularity formation [6]. Moreover, a small amount of angular momentum does not prevent the occurrence of a singularity when a Kerr black hole forms.
In this paper we consider spheroids in which the particles have angular momentum about the symmetry axis. We choose equal numbers of corotating and counterrotating particles, so that the configuration has no net angular momentum. This choice simplifies the gravitational field equations, while retaining the centrifugal barrier for particles approaching the axis. The resulting set of field equations is identical to the ones solved previously $[1,2]$, except for the presence of simple modifications in the matter source terms arising from the angular motion of the particles.

In the nonrotating cases described previously, the spindle singularities were similar to the naked singularity formed by the collapse of a nonrotating infinite cylinder of particles [4,7]. The fate of an infinite cylinder with equal numbers of corotating and counterrotating particles is therefore interesting. Recently, Apostolatos and Thorne [8] have shown analytically that in the case of a single cylindrical shell of such oppositely directed particles, the collapse must be halted at a finite radius and hence a spindle singularity cannot form. Thus the relativistic behavior is qualitatively the same as the Newtonian for this idealized example. Does this result also hold for relativistic systems of finite length? Our simulations address the effect of angular momentum on a collection of particles that is bounded in an asymptotically flat spacetime.

\section{NEWTONIAN ANALOGUE}

In the Newtonian limit, the configurations that we evolve are homogeneous spheroids of collisionless corotating and counterrotating particles. The dynamical behavior of such systems is well understood when the angular velocity has a constant magnitude throughout the configuration. In this case, the spheroid remains homogeneous and spheroidal in shape. Hence the equations of 
motion [9] reduce to those of particles on the surface at the equator and on the pole. To see why angular momentum inevitably halts the collapse, one merely invokes energy and angular momentum conservation.

Let $a$ denote the equatorial radius of the spheroid and $c$ the polar radius. If $a<c$, the spheroid is prolate and we define the eccentricity by

$$
e \equiv\left(1-a^{2} / c^{2}\right)^{1 / 2}
$$

If $a>c$ then the spheroid is oblate and we interchange $a$ and $c$ in the definition of $e$. If all the particles in the spheroid are rotating in the same direction about the symmetry axis with angular velocity $\Omega$, then the total angular momentum of the spheroid is

$$
J_{+}=I \Omega \text {, }
$$

where the moment of inertia is

$$
I=\frac{2}{5} M a^{2}
$$

and $M$ is the mass. If $\chi$ is the fraction of particles that orbit in the $+\phi$ direction, with the remainder orbiting in the opposite direction, then the spheroid has a net angular momentum

$$
J=(2 \chi-1) J_{+} \cdot
$$

Both $J$ and $J_{+}$are conserved during the evolution. Moreover, in Newtonian theory the evolution is independent of the value of $\chi$, whereas in general relativity the solution depends on the net angular momentum (e.g., dragging of inertial frames).

The rotational kinetic energy is

$$
T_{\text {rot }}=\frac{J_{+}^{2}}{2 I},
$$

while the total kinetic energy is

$$
T=\frac{1}{10} M\left(2 \dot{a}^{2}+\dot{c}^{2}\right)+T_{\text {rot }} .
$$

The potential energy is

$$
W= \begin{cases}-\frac{3}{5} \frac{M^{2}}{a} \frac{\arcsin e}{e} & \text { (oblate) } \\ -\frac{3}{10} \frac{M^{2}}{c e} \ln \frac{1+e}{1-e} & \text { (prolate) }\end{cases}
$$

(Here and throughout we set Newton's gravitational constant and the speed of light equal to one.) The total energy is conserved:

$$
E=T+W=\text { const . }
$$

From the above equations, it is clear that a configuration with a nonzero $T_{\text {rot }}$ cannot collapse to a prolate spindle singularity with $a \rightarrow 0, c \rightarrow$ const, and $e \rightarrow 1: T_{\text {rot }} \rightarrow+\infty$ like $1 / a^{2}$, while $W \rightarrow-\infty$ like $\ln a$.

One characteristic of a spindle singularity is that the equations of motion [9] cannot be integrated through it because they have divergent terms. A second feature of such a singularity is that the gravitational tidal field blows up in its vicinity. As a measure of the gravitational tidal field, consider the tidal tensor in Cartesian coordinates:

$$
\Phi_{i j} \equiv \frac{\partial^{2} \Phi}{\partial x_{i} \partial x_{j}} .
$$

From this quantity, construct the simple scalar function

$$
I^{N}=8 \Phi_{i j} \Phi_{i j}
$$

where summation over repeated indices is implied. With the factor of 8 , this quantity is the Newtonian limit of the Riemann invariant

$$
I \equiv R_{\alpha \beta \gamma \delta} R^{\alpha \beta \gamma \delta}
$$

evaluated in vacuum. In the highly eccentric limit $e \rightarrow 1$, the tidal invariant for a homogeneous prolate spindle has the following singular limiting behavior just above the pole in the vacuum exterior:

$$
I_{\text {pole }}^{N} \rightarrow \frac{108}{c^{6}} \frac{M^{2}}{\left(1-e^{2}\right)^{2}} .
$$

The corresponding behavior in the vacuum just outside the equator, and everywhere in the interior, is

$$
I_{\text {interior }}^{N} \rightarrow I_{\mathrm{eq}}^{N} \rightarrow \frac{1}{3} I_{\text {pole }}^{N} .
$$

This singularity for a prolate spindle is in contrast with the tidal field for a homogeneous sphere of radius $R$, for which

$$
I^{N}=\left\{\begin{array}{cl}
\frac{48 M^{2}}{r^{6}} & \text { (exterior) } \\
\frac{24 M^{2}}{R^{6}} & \text { (interior) }
\end{array}\right.
$$

which is bounded everywhere.

\section{GENERAL-RELATIVISTIC CASE}

We have followed the collapse of collisionless spheroids of rotating particles in full general relativity. The field equations are expressed in $3+1$ form following Arnowitt, Deser, and Misner [10]. We use maximal time slicing and isotropic spatial coordinates. The metric is

$$
\begin{aligned}
d s^{2}= & -\alpha^{2} d t^{2}+A^{2}\left(d r+\beta^{r} d t\right)^{2} \\
& +A^{2} r^{2}\left(d \theta+\beta^{\theta} d t\right)^{2}+B^{2} r^{2} \sin ^{2} \theta d \phi^{2} .
\end{aligned}
$$

The basic code is identical to the one described in Refs. [1] and [2].

The matter consists of equal numbers of corotating and counterrotating particles. Each particle is assigned initial four-velocity components

$$
u_{r}=0, u_{\theta}=0, u_{\phi}= \pm \xi r^{2} \sin ^{2} \theta \Omega \text {. }
$$

Here the angular velocity is

$$
\Omega=\left(\frac{3 A_{1}}{2 a^{2} c}\right)^{1 / 2},
$$

where 


$$
A_{1}=\frac{1}{e^{2}}-\frac{1-e^{2}}{2 e^{3}} \ln \frac{1+e}{1-e},
$$

and $\xi$ is a constant. When $\xi=1$, the velocity $u_{\phi}$ is the value at which centrifugal force balances gravity in the Newtonian limit. When $\xi=0$ all the particles are initially at rest, and since $u_{\phi}$ is conserved, there is never any angular motion. This is the case studied in Refs. [1] and [2]. In this paper we consider cases in which $0 \leq \xi \lesssim 1.5$.

Because there is no net angular momentum, the initial-value equations in general relativity are essentially identical to the nonrotating case solved in Ref. [11]. The only difference is the presence of a $\gamma$ factor in the density source term for the Hamiltonian constraint because of the rotational velocity. Accordingly, the momentarily static solution given in Sec. III of Ref. [11] is unchanged provided we restore $\rho^{*}$ in place of $\rho$ in Eqs. (3.5) and (3.7) of that paper. Also, Eq. (3.10) for the rest mass now becomes

$$
M_{0}=\int \frac{\rho^{*}}{\gamma} \psi^{6} d^{3} x
$$

where

$$
\gamma=\left(1+\frac{u_{\phi}^{2}}{B^{2} r^{2} \sin ^{2} \theta}\right)^{1 / 2}
$$

The metric is determined analytically following the prescription in Ref. [11]. The rest-mass density is now given by

$$
\rho=\rho^{*} / \gamma^{2}
$$

Having specified the particle velocity and density profiles, we can now distribute particles that sample the initial phase-space distribution. In the Newtonian limit, the configurations are homogeneous spheroids, but relativistic configurations are inhomogeneous spheroids with density typically increasing outwards.

As in Refs. [1] and [2], typical simulations were performed with a spatial grid of 100 radial and 32 angular zones, and with 6000 test particles. Again, a key feature enabling us to snuggle close to singularities was that the angular grid could fan and the radial grid could contract to follow the matter.

In Refs. [1] and [2] we followed the evolution of a prolate spheroid with initial semimajor axis $c=10 M$, eccentricity $e=0.9$, and $\xi=0$ (no rotation). This configuration collapses to a spindle singularity near the pole without the appearance of an apparent horizon. The spindle consists of a concentration of matter near the axis at $r=5 \mathrm{M}$. The value of the Riemann invariant $I$ rises without bound in a broad region near the pole and extending out into the vacuum. The maximum value of $I$ determined by our code is limited only by the resolution of the angular grid: The better we resolve the spindle, the larger the value of $I$ we can attain before the singularity causes the code (and spacetime) to break down. The absence of an apparent horizon makes this example a strong candidate for a naked singularity. Moreover, the evolution of this configuration is typical of the evolution found for all nonrotating prolate spheroids with large semimajor axes.
Only for highly relativistic spheroids with moderately small initial semimajor axes $(c \lesssim 3 M)$ did the collapse produce a black hole.

To assess the role of rotation, we here consider prolate spheroids with the same initial values of $c$ and $e$ as the example described above, but with various nonzero values of the rotation parameter $\xi$. We are only interested in small enough values of $\xi$ that the spheroid still implodes in the equatorial plane $(\xi \lesssim 1.2)$. For sufficiently large values of $\xi$ in this range, we find that the evolution is significantly modified by rotation. For $\xi \gtrsim 0.4$, the spheroid unambiguously collapses to a black hole. For the case $\xi=0.9$, Fig. 1 shows the mean equatorial and polar radii, defined by summing over particle positions:

$$
\begin{aligned}
& a_{\mathrm{rms}} \equiv\left[\frac{5}{2 N_{p}} \sum_{j} r_{j}^{2} \sin ^{2} \theta_{j}\right]^{1 / 2}, \\
& c_{\mathrm{rms}} \equiv\left[\frac{5}{N_{p}} \sum_{j} r_{j}^{2} \cos ^{2} \theta_{j}\right]^{1 / 2},
\end{aligned}
$$

where $N_{p}$ is the total number of particles. The numerical factors in Eq. (3.8) have been chosen so that these quantities will be exactly equal to the semiaxes for a uniform density spheroid. Also shown in Fig. 1 are the corresponding values of these radii obtained by integration of the Newtonian spheroidal equations of motion [9]. By contrast with the nonrotating relativistic spheroid, we see that centrifugal forces here are sufficient to hold back the formation of a spindle. While the equatorial axis is held back, the polar axis collapses unimpeded, until the configuration becomes almost spherical and implodes to a black hole. The Newtonian configuration, on the other hand, oscillates homologously forever, also without forming a spindle singularity.

An alternative way to characterize the amount of rota-

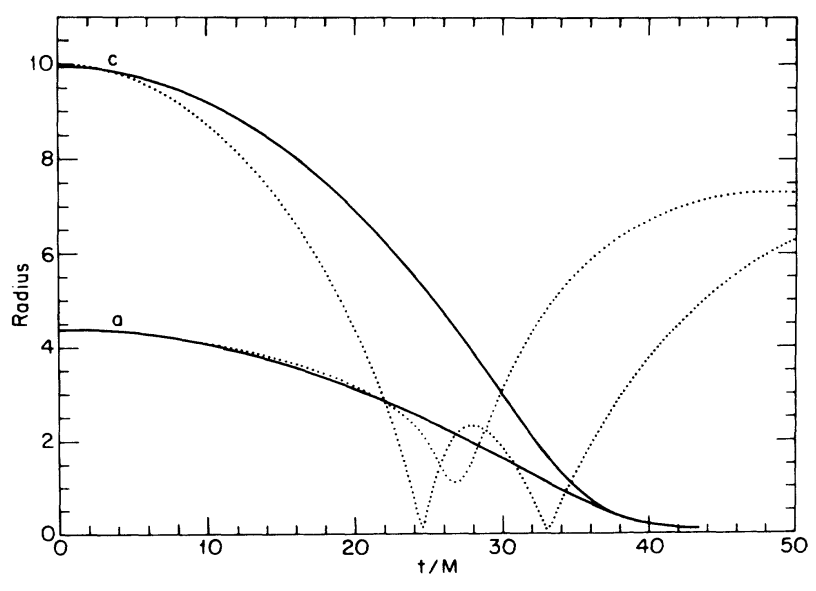

FIG. 1. Mean equatorial and polar radii as functions of time for a rotating spheroid with $\xi=0.9$. The quantity $a$ is the mean equatorial radius and $c$ is the polar radius, both in units of $M$. The initial configuration is prolate with $c=10$ and eccentricity 0.9 . Solid lines show the relativistic evolution, dotted lines the Newtonian evolution. The relativistic spheroid collapses to a black hole, while the Newtonian spheroid oscillates indefinitely. 
tion in the cluster is to define the relativistic analogue of the Newtonian $J_{+}$of Eq. (2.2):

$$
J_{+}=\sum_{j} m_{j}\left|u_{\phi}\right|_{j}
$$

The significance of this parameter is that, if all the particles were rotating with the same sense, they could all collapse to form a Kerr black hole only if $a_{+} / M \equiv J_{+} / M^{2}$ is less than 1. No such restriction applies for the cases explored here, since the net angular momentum is zero.

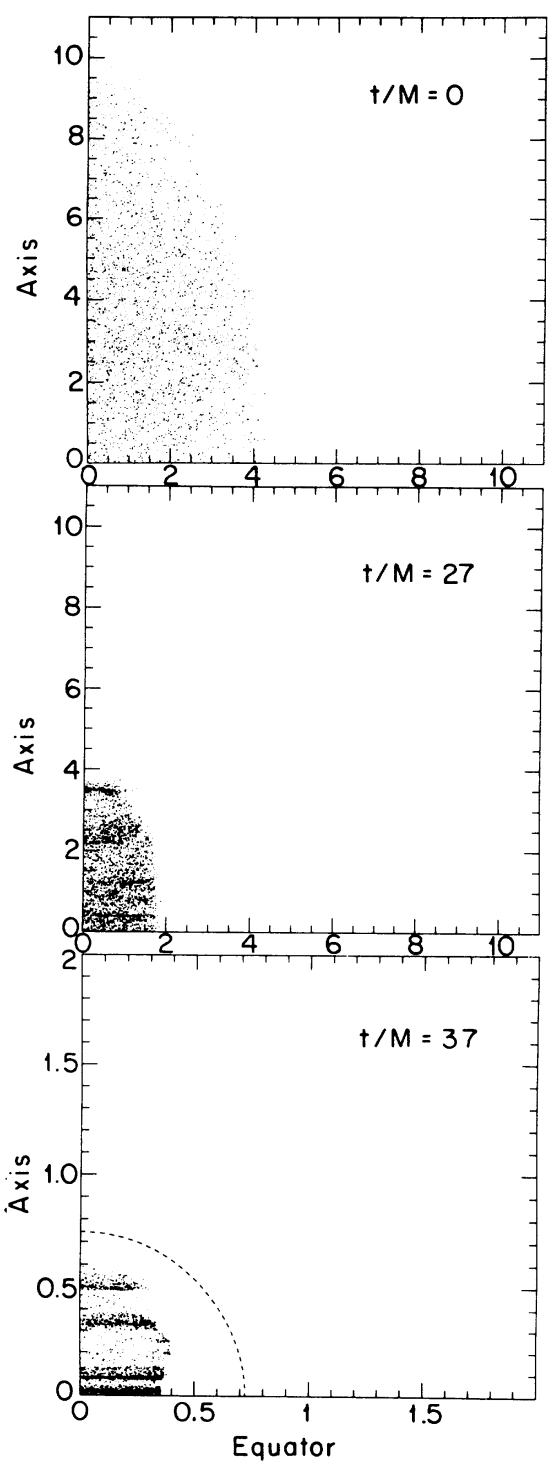

FIG. 2. Snapshots of the particle positions at selected times for the case shown in Fig. 1. The coordinate positions (in units of $M$ are projected onto a meriodional plane. The collapse proceeds nonhomologously and results in the formation of a black hole. The apparent horizon that forms at late times is shown by the dashed line. We have expanded the scale in the final frame to zoom in on the black hole. Note the growth of axisymmetric perturbations (sheets perpendicular to the symmetry axis) arising from discreteness in the particle distribution.
For the configuration in Fig. $1, a_{+} / M=0.62$.

Snapshots of the particle positions at selected times are shown in Fig. 2. By the time we terminate the calculation, virtually all of the mass is inside the black hole. The apparent horizon is nearly spherical and has an area $\mathcal{A} / 16 \pi M^{2}$ close to the asymptotic theoretical limit of 1 . Gravitational waves carry away a negligible fraction $(<1 \%)$ of the total mass-energy.

For larger initial semimajor axis, the final fate is the same but the evolutionary track is different. Figure 3 shows the evolution of the mean axes for collapse from $20 M$ with the same initial eccentricity and $\xi=1.2$. Here $a_{+} / M=1.1$. In this case the configuration pancakes during the implosion, overshoots, becomes prolate again, and then collapses to a spherical black hole. Snapshots of the particle positions for this case are shown in Fig. 4.

The contrast between collapse with $\xi=0$ and $\xi$ appreciable is quite stark: with no rotation, a spindle singularity forms, while with large rotation, collapse leads to the formation of a black hole. However, the key question is, does any angular momentum, however small, prevent the formation of a spindle singularity outside an apparent horizon? Figures 5-8 show the collapse of the same configuration shown in Fig. 1, but with $\xi=0.1$, $a_{+} / M=0.069$. For comparison we also show the results for no rotation [12], $\xi=0$. Even with rotation, it appears that the spheroid is collapsing to a spindle singularity near the pole, without the appearance of an apparent horizon. In fact, to the accuracy of our code, we cannot distinguish the behavior of the cases with $\xi=0$ and $\xi=0.1$. Even when we increase our computational resources, e.g., doubling the angular grid to snuggle closer to the spindle, the dynamical behaviors are still essentially indistinguishable.

With a finite number of particles and finite grid resolution, small fluctuations in the numerical results do not

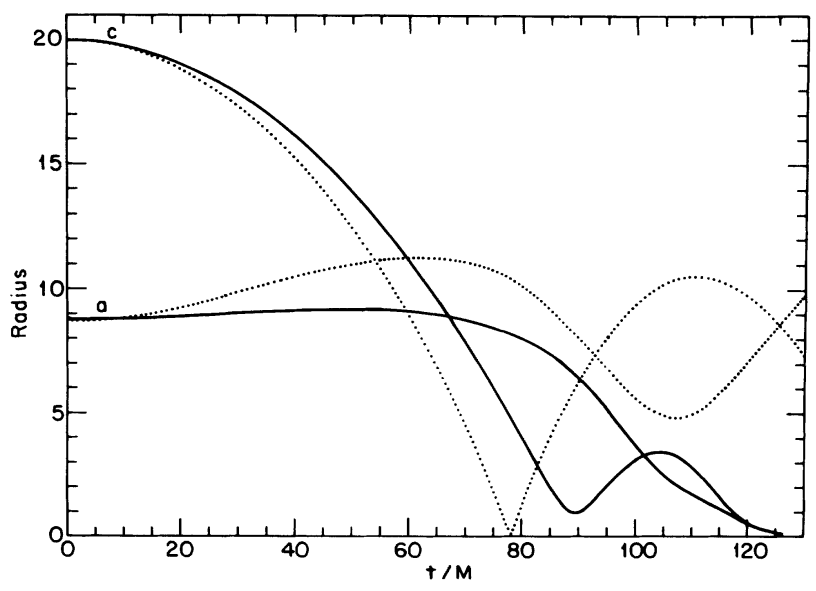

FIG. 3. Mean equatorial and polar radii as functions of time for a rotating spheroid with $\xi=1.2$. The initial configuration is prolate with $c=20$ and eccentricity 0.9 . Labels are the same as in Fig. 1. The relativistic spheroid collapses to a pancake, overshoots, becomes prolate, and then implodes to a black hole. By contrast, the Newtonian spheroid oscillates indefinitely. 
necessarily constitute physical effects. By experimenting with changes in computational resources, we can distinguish which features are significant. In particular, the appearance of a spike in $I$ at the pole with a height much bigger than the exterior Schwarzschild value $48 M^{2} / r_{S}^{6}$ signifies a spindle singularity. (Here $r_{S}$ is the

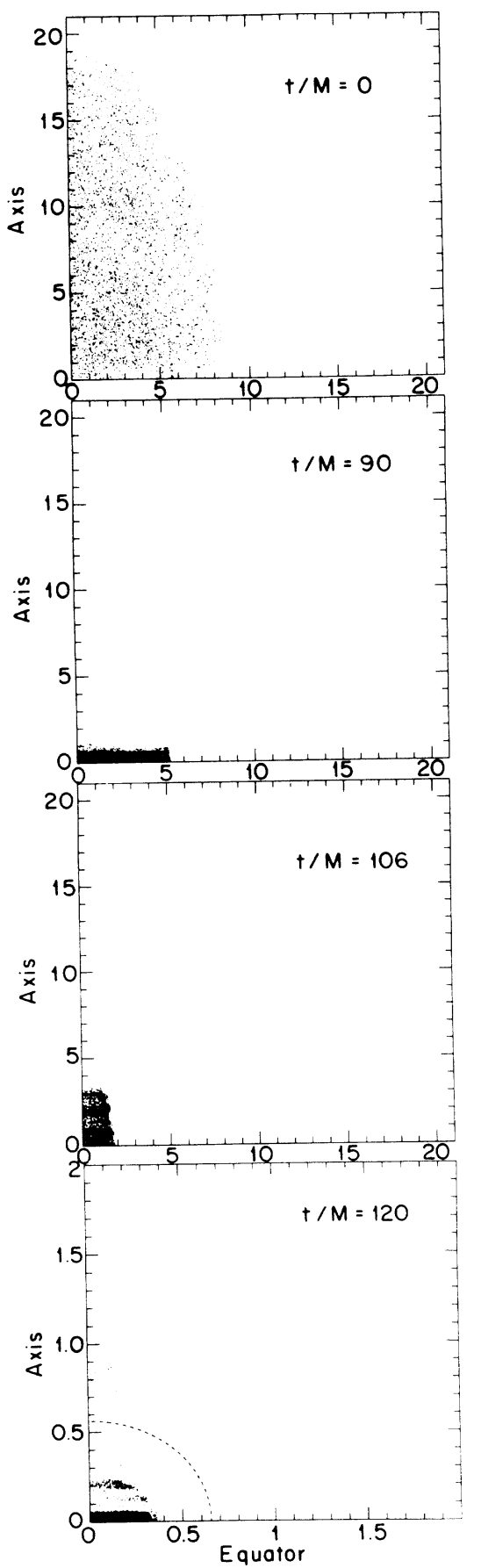

FIG. 4. Snapshots of the particle positions at selected times for the case shown in Fig. 3. Labels are the same as in Fig. 2. Again we expand the scale in the final frame to zoom in on the black hole.
Schwarzschild radial coordinate.) The precise peak value of $I$ attained before the code loses accuracy is not a meaningful quantity. For these reasons we believe that Figs. 7 and 8 are exhibiting the same singular behavior. Moreover, with increased resolution, we do not see the peak value of $I$ asymptoting to a finite value, but rather increasing without bound for both $\xi=0$ and $\xi=0.1$. In the case of a rotating spheroid in Newtonian physics, in which the particles avoid the axis, $I$ would reach a finite maximum value when the eccentricity reaches its maximum [cf. Eqs. (2.12) and (2.13)], but would not blow up to infinity.

We have also run cases with $\xi \leq 0.05$, and these are totally indistinguishable from the nonrotating case. It appears that $\xi \approx 0.1$ is the critical value below which collapse leads to a spindle singularity without the appearance of an apparent horizon.
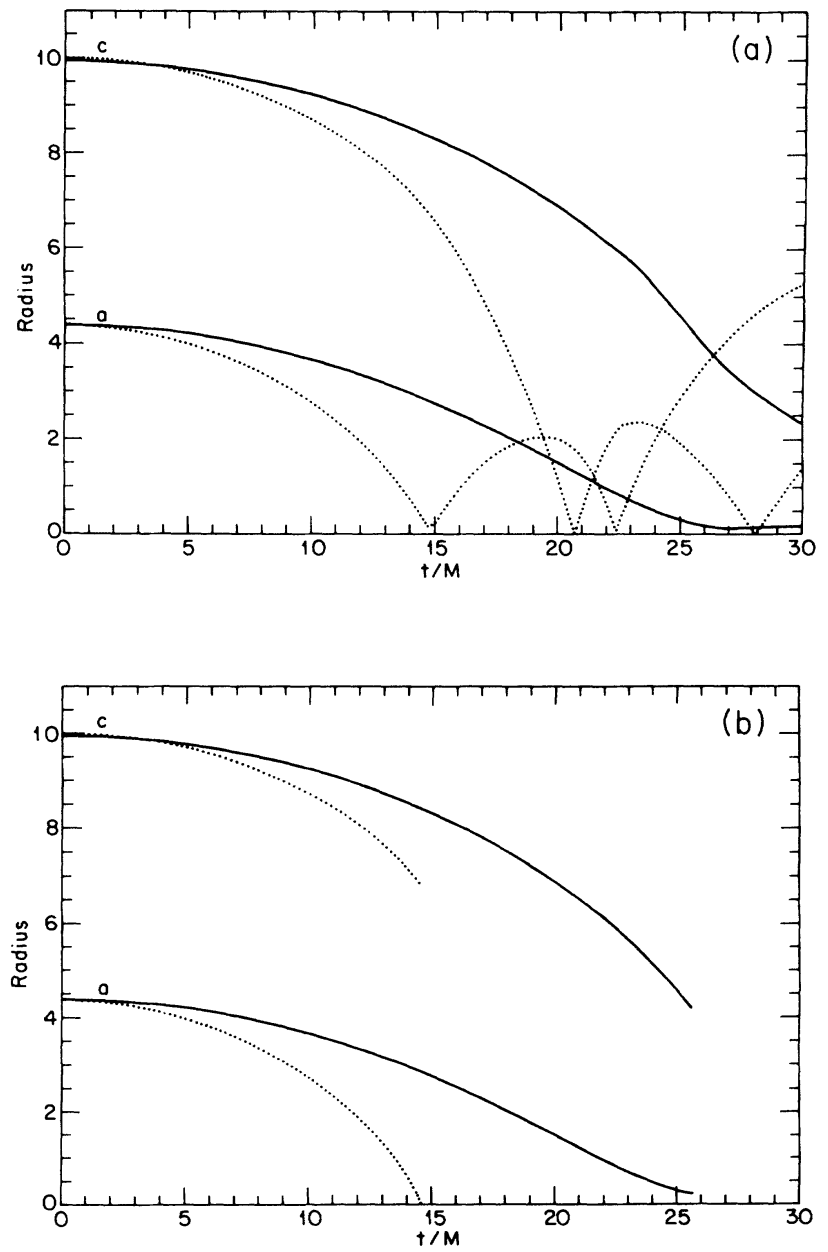

FIG. 5. Mean equatorial and polar radii as functions of time for a prolate spheroid with rotation parameter (a) $\xi=0.1$ and (b) $\xi=0$. Both configurations have the same initial size and shape as the case shown in Fig. 1. The relativistic curves in (a) and (b) are almost indistinguishable, and the spheroids evolve to spindle singularities with no apparent horizons. By contrast, rotation prevents a spindle singularity in the Newtonian case. 

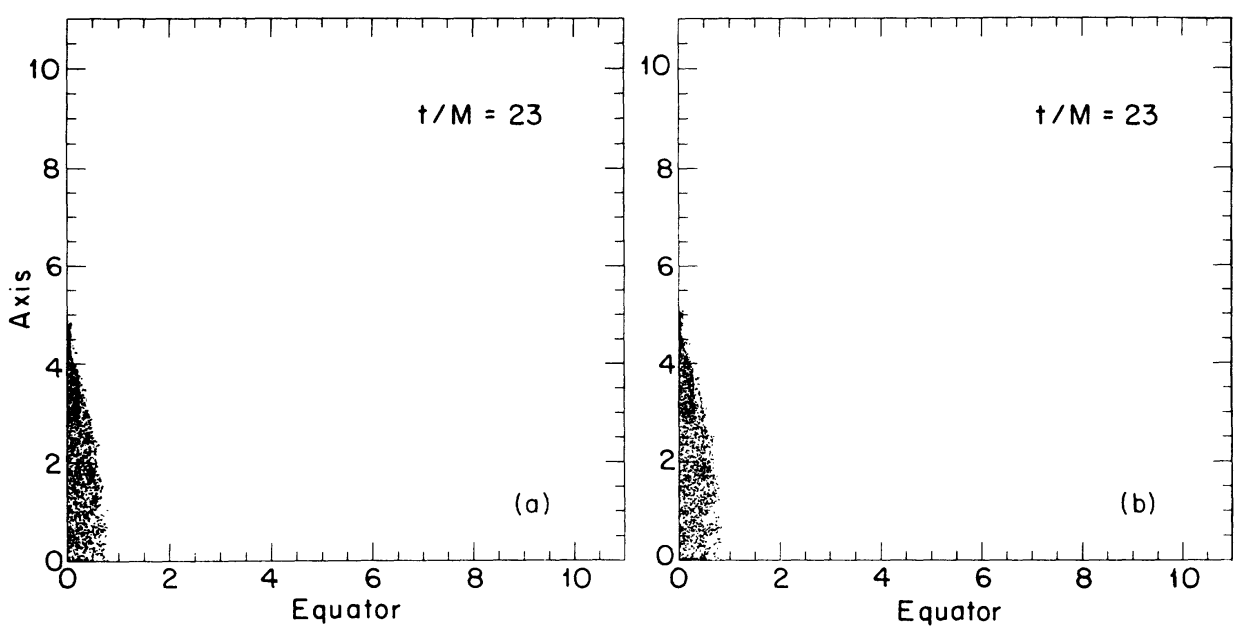

FIG. 6. Snapshots of the particle positions at the final times for the cases shown in Figs. 5(a) and $5(\mathrm{~b})$. The particle distributions are virtually indistinguishable, and a spindle singularity forms on the axis with no apparent horizon to terminate the collapse.

\section{DISCUSSION}

Our numerical simulations demonstrate that, as expected, for appreciable angular momentum the formation of a spindle singularity during gravitational collapse is prevented. However, for small angular momentum our numerical simulations cannot distinguish rotating from nonrotating collapse, where a spindle singularity appears without an apparent horizon. This behavior is in contrast with the Newtonian result, and also with the case of relativistic infinite cylinders [8], where no spindle singularity can occur when there is rotation. Accordingly, our simulations are unable to rule out the possibility that asymptotically flat configurations with small, but nonzero, angular momentum can collapse to form spindle singularities. If this possibility occurs, then even prolate spheroids with angular momentum are good candidates for naked singularities.

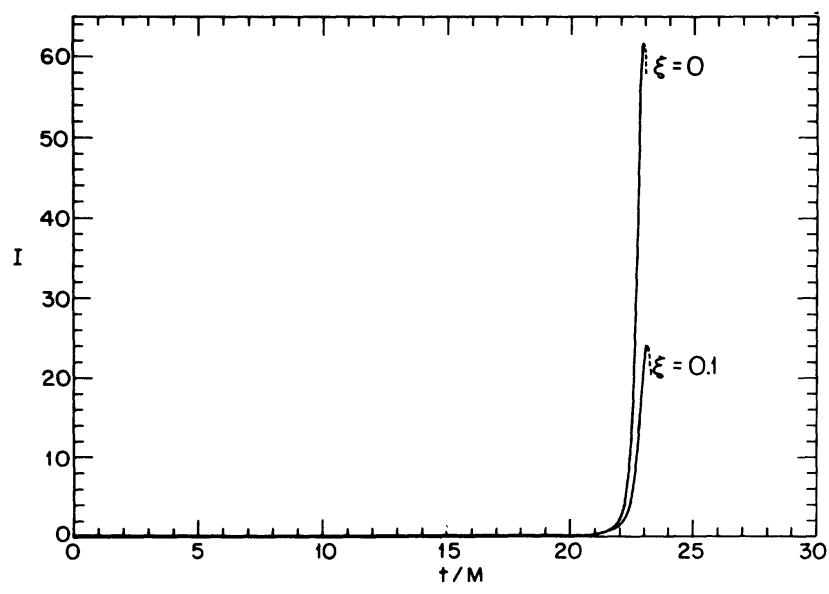

FIG. 7. Growth of the Riemann invariant $I$ (in units of $M^{-4}$ ) vs time for the cases shown in Figs. 5(a) and 5(b). The invariant $I$ is measured in the vacuum exterior just above the pole. We use dots to show where the singularities have caused the code to become inaccurate. Increasing the number of angular grid points causes the peak values to grow, apparently without bound.
Of course, it is possible that the situation might be different for configurations with a small net angular momentum, which would be the case in our simulations if all the particles rotated in the same direction. Unlike in Newtonian theory, the gravitational field is different when there is a net angular momentum, and so it is difficult to guess the outcome. We do know that a small net angular momentum does not prevent the formation of a singularity in gravitational collapse to a Kerr black hole. On the other hand, the character of the singularity in a Kerr black hole does differ from that in a Schwarzschild black hole. We hope to study configurations with net angular momentum in the future.

One potential improvement to our numerical treatment will be to switch from spherical polar to cylindrical coordinates. In cylindrical coordinates we will be able to probe the singular region near the axis with far better resolution for the same computational resources. We originally adopted spherical polar coordinates to treat the outgoing radiation at large distances accurately. Because this radiation has turned out to be small, we do not foresee any difficulties when we implement cylindrical coordinates.

We decide whether or not a given singularity is naked by whether there is an apparent horizon. As pointed out in Refs. [1] and [2], the absence of an apparent horizon

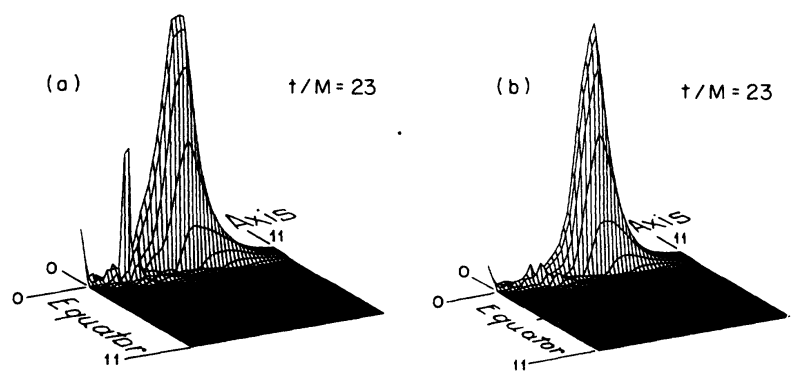

FIG. 8. Profile of $I$ in a meridional plane for the cases shown in Figs. 5(a) and 5(b). For the case of 32 angular zones shown here, the peak value of $I$ is $31 / M^{4}$ for case (a) and $54 / M^{4}$ for case (b). It occurs on the axis just outside the matter. 
does not necessarily imply the absence of a global event horizon, although the converse is true. Because singularities cause our numerical integrations to terminate, we cannot map out a spacetime arbitrarily far into the future, which would be necessary to completely rule out the formation of an event horizon. A second potential improvement to the present investigation thus would be to find a time slicing that allows the time integrations to continue further into the future in the region away from the axis, while avoiding the singularity on the axis. Continued failure to find an apparent horizon, or outgoing light rays that turn around, would strengthen the argument that the singularity is naked.

The cosmic-censorship hypothesis [13] states that singularities formed during collapse will always be clothed by event horizons, and hence can never be visible from the outside. If in fact collapsing spheroids can form naked singularities even with small angular momentum, which is consistent with the simulations reported here, this would place severe restrictions on the validity of the unqualified cosmic-censorship hypothesis.

\section{ACKNOWLEDGMENTS}

We thank A. Abrahams, H. Apostalatos, and K. Thorne for stimulating discussions. This research was supported in part by NSF Grants Nos. AST 90-15451 and PHY 90-07834 and NASA Grant No. NAGW-2364 at Cornell University. Computations were performed on the Cornell National Supercomputer Facility.
[1] S. L. Shapiro and S. A. Teukolsky, Phys. Rev. Lett. 66, 994 (1991).

[2] S. L. Shapiro and S. A. Teukolsky, Am. Sci. 79, 330 (1991).

[3] C. C. Lin, L. Mestel, and F. H. Shu, Astrophys. J. 142, 1431 (1965).

[4] K. S. Thorne, in Magic Without Magic: John Archibald Wheeler, edited by J. Klauder (Freeman, San Francisco, 1972), p. 1

[5] See E. Horst, Math. Methods Appl. Sci. 4, 19 (1982), for a formal proof; see [9] for a numerical demonstration.

[6] For a formal proof for collisionless collapse, see A. D. Rendall, Class. Quantum Grav. (to be published).

[7] C. W. Misner, K. S. Thorne, and J. A. Wheeler, Gravitation (Freeman, San Francisco, 1973), p. 867.
[8] H. Apostolatos and K. S. Thorne (unpublished).

[9] S. L. Shapiro and S. A. Teukolsky, Astrophys. J. 318, 542 (1987).

[10] R. Arnowitt, S. Deser, and C. W. Misner, in Gravitation: an Introduction to Current Research, edited by L. Witten (Wiley, New York, 1962), p. 227.

[11] T. Nakamura, S. L. Shapiro, and S. A. Teukolsky, Phys. Rev. D 38, 2972 (1988).

[12] Small differences between the plots shown here for the nonrotating case and those in Refs. [1] and [2] result from minor technical improvements to the code.

[13] R. Penrose, Riv. Nuovo Cimento 1 (Numero Special), 252 (1969). 


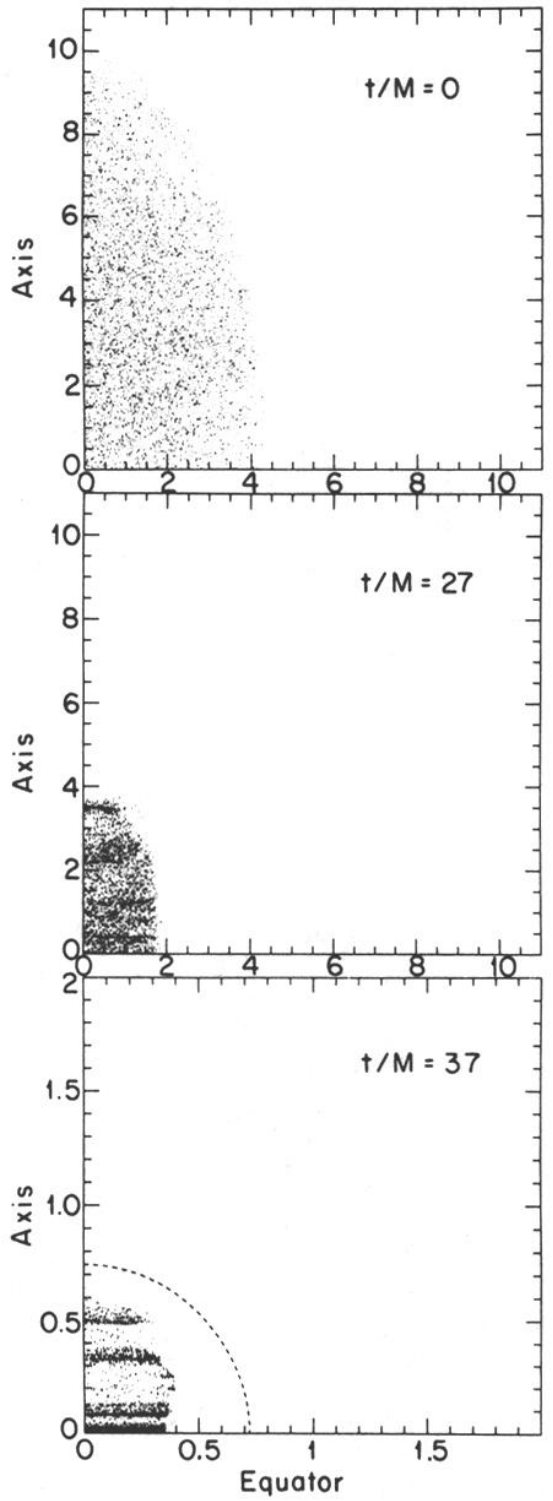

FIG. 2. Snapshots of the particle positions at selected times for the case shown in Fig. 1. The coordinate positions (in units of $M$ ) are projected onto a meriodional plane. The collapse proceeds nonhomologously and results in the formation of a black hole. The apparent horizon that forms at late times is shown by the dashed line. We have expanded the scale in the final frame to zoom in on the black hole. Note the growth of axisymmetric perturbations (sheets perpendicular to the symmetry axis) arising from discreteness in the particle distribution. 


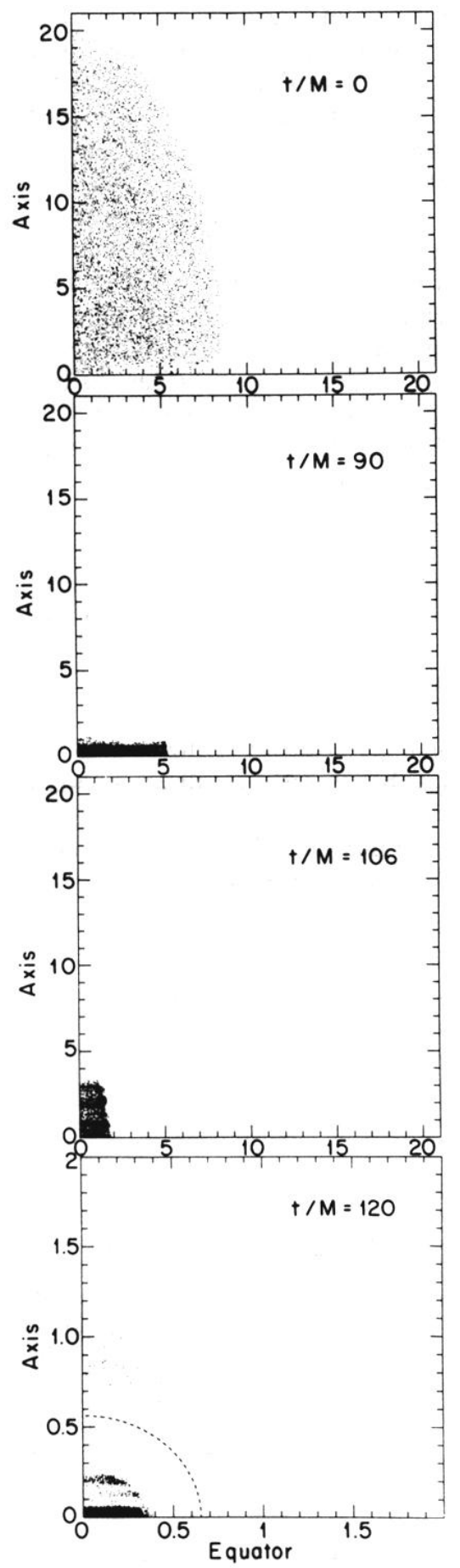

FIG. 4. Snapshots of the particle positions at selected times for the case shown in Fig. 3. Labels are the same as in Fig. 2. Again we expand the scale in the final frame to zoom in on the black hole. 

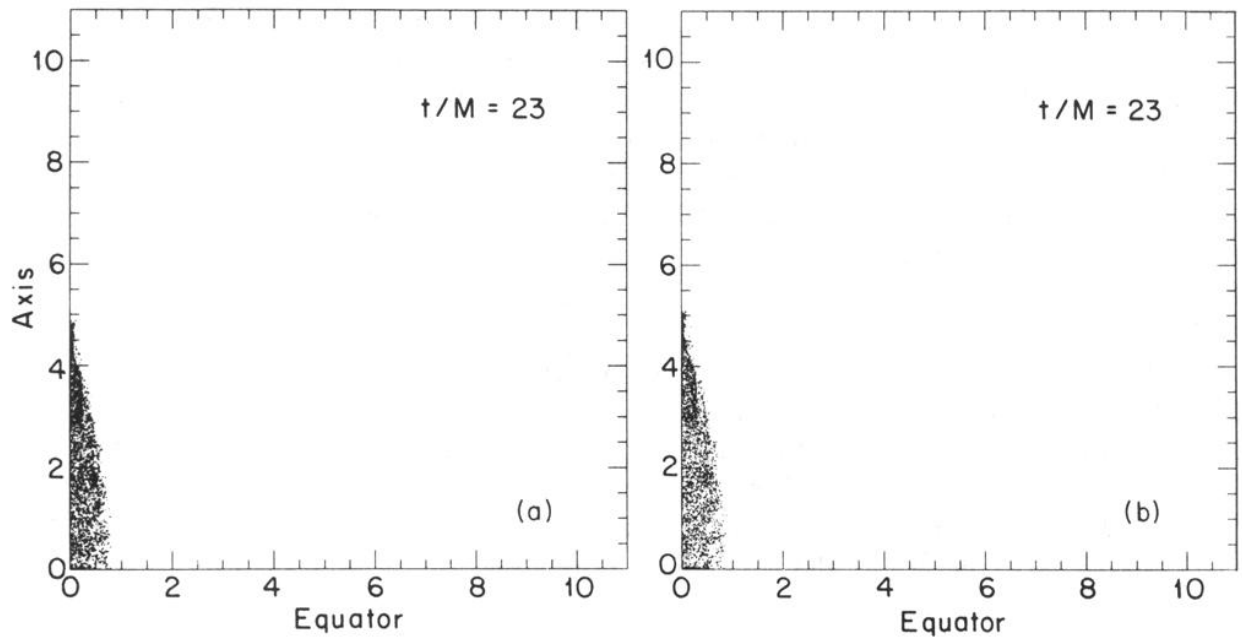

FIG. 6. Snapshots of the particle positions at the final times for the cases shown in Figs. 5(a) and 5(b). The particle distributions are virtually indistinguishable, and a spindle singularity forms on the axis with no apparent horizon to terminate the collapse. 

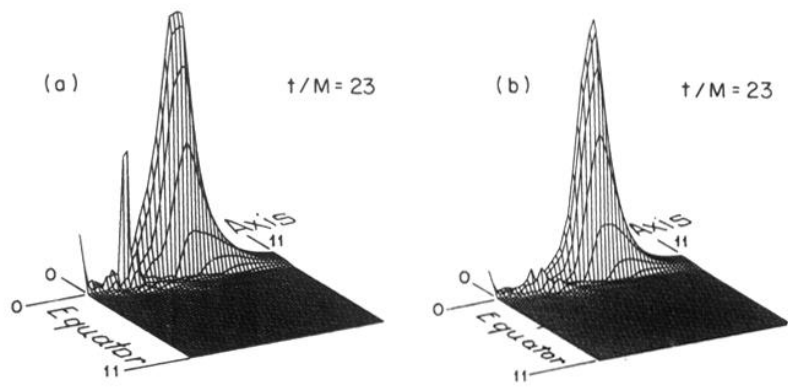

FIG. 8. Profile of $I$ in a meridional plane for the cases shown in Figs. 5(a) and 5(b). For the case of 32 angular zones shown here, the peak value of $I$ is $31 / M^{4}$ for case (a) and $54 / M^{4}$ for case (b). It occurs on the axis just outside the matter. 